\title{
Gestión de las redes sociales en las campañas electorales españolas en 2019
}

\author{
Juan Enrique Gonzálvez Vallés \\ Almudena Barrientos-Báez \\ David Caldevilla-Domínguez \\ Universidad Complutense de Madrid \\ jegonzalvez@ucm.es \\ almbarri@ucm.es \\ davidcaldevilla@ccinf.ucm.es
}

Fecha de presentación: agosto de 2020

Fecha de aceptación: enero de 2021

Fecha de publicación: diciembre de 2021

Cita recomendada: GonZÁlveZ VAllÉs, J. E.; BARRIENTOS-BÁEZ, A. y CALDEVILLA-DOMíNGUEZ, D. (2021). "Gestión de las redes sociales en las campañas electorales españolas en 2019». Anàlisi: Quaderns de Comunicació i Cultura, 65, 67-86. DOI: <https://doi.org/10.5565/rev/ analisi.3347>

\section{Resumen}

El objeto de estudio de este trabajo es el análisis de la estrategia de comunicación que llevan a cabo los partidos políticos españoles durante las distintas convocatorias electorales en el año 2019 en las redes sociales. De forma detallada se analiza la actividad, la capacidad para generar sentimiento de pertenencia y las distintas formas de interacción de estas formaciones, así como los formatos más exitosos. Partimos de la hipótesis de que los partidos políticos que están presentes tanto en todas las elecciones como en todas las circunscripciones electorales pueden lograr un mayor rendimiento de su presencia en los medios sociales. Como técnica metodológica, se propone el análisis de redes sociales (ARS) a los perfiles de Facebook, Twitter e Instagram de estos actores para la obtención de datos tanto cuantitativos como cualitativos. En total, se realiza el análisis de la distribución de 2.357.039 fanes en Facebook, 3.727.197 seguidores en Twitter y 919.731 en Instagram. Igualmente, se analizan 9.938 publicaciones que generan un engagement promedio de 7.066,92. Las conclusiones dejan a Vox y Unidas Podemos como los partidos más ciberactivos y que más movilización producen a través de las redes sociales.

Palabras clave: redes sociales; campańas; elecciones; seguidores; publicaciones; engagement

Resum. Gestió de les xarxes socials a les campanyes electorals espanyoles el 2019

L'objecte d'estudi d'aquest treball és l'anàlisi de l'estratègia de comunicació que van fer els partits polítics espanyols durant les diferents convocatòries electorals l'any 2019 a 
les xarxes socials. S'analitza de manera detallada l'activitat, la capacitat per generar sentiment de pertinença i les diferents formes d'interacció d'aquestes formacions, així com els formats més reeixits. Partim de la hipòtesi que els partits polítics que són presents tant a totes les eleccions com a totes les circumscripcions electorals poden aconseguir un rendiment més alt de la seva presència als mitjans socials. Com a tècnica metodològica, es proposa l'anàlisi de xarxes socials (ARS) als perfils de Facebook, Twitter i Instagram d'aquests actors per obtenir dades tant quantitatives com qualitatives. En total, es fa l'anàlisi de la distribució de 2.357.039 fans a Facebook, 3.727.197 seguidors a Twitter i 919.731 a Instagram. Igualment, s'analitzen 9.938 publicacions que generen un compromís mitjà de 7.066,92 seguidors. Les conclusions deixen Vox i Unides Podemos com els partits més ciberactius i que més mobilització produeixen a través de les xarxes socials.

Paraules clau: xarxes socials; campanyes; eleccions; seguidors; publicacions; compromís

\section{Abstract. Management of social media in Spanish electoral campaigns in 2019}

This work analyses the communication strategies on social networks by Spanish political parties during the various elections of 2019. It analyses in detail their activity, their ability to generate a feeling of belonging and the different forms of interaction of these formations, and the most successful formats. We introduce the hypothesis that political parties that are present in all elections and in all electoral constituencies can achieve a higher return on their social media presence. As a methodological technique, the study undertakes an analysis of social networks (ASN) for the Facebook, Twitter and Instagram accounts of these actors, to obtain both quantitative and qualitative data. In total, it analyses the distribution of 2,357,039 fans on Facebook; 3,727,197 followers on Twitter; and 919,731 followers on Instagram. Likewise, 9,938 publications are analysed, which generate an average of 7,066.92 engagements. The conclusions show Vox and Unidas Podemos as the most cyber-active parties and the ones which achieve the most mobilisation through social networks.

Keywords: social networks; campaigns; elections; followers; publications; engagement

\section{Introducción}

La comunicación política en campaña electoral conlleva efectos particulares en diferentes redes sociales, empezando por el significativo aumento del consumo de noticias (Samuel-Azran y Hayat, 2019: 72) y su empleo extensivo por parte de los más jóvenes (Catalina-García et al., 2019). Destacan los estudios de Casero (2018), que pone el foco principal sobre los actores intervinientes y los efectos que las redes suponen para la opinión pública y publicada:

Las redes sociales amplían el número y tipo de actores que interactúan y negocian en el campo de la comunicación política. De un escenario marcado, casi exclusivamente, por las relaciones entre periodistas y políticos estamos pasando a un panorama más abierto y descentralizado en el que un mayor número de actores participan de los intercambios que contribuyen a definir la esfera pública gracias a las plataformas digitales. Esto afecta decisivamente a la producción de información política. (p. 967) 
Como argumentan Feijoo y Guerrero (2018), las TIC permiten que los públicos sean los que revelen sus hábitos e intereses al mercado. La interactividad a través de Twitter y Facebook facilita el comentario y la opinión minuto a minuto, con lo que los protagonistas están obligados a dar lo mejor de sí en cada intervención (Barrientos-Báez et al., 2019). Las redes son importantes para la comunicación política, porque, como dice Grández (2016) sobre la televisión, son baratas y accesibles. Asimismo, se puede delimitar su ámbito de actuación dentro del marco geográfico, puesto que, aunque la utilización de las redes sociales se inicia con la campaña de Barack Obama en 2008 en Estados Unidos y su estudio deja importantes observaciones (Woolley et al., 2010), posteriormente, en España, también se asume este nuevo reto para tratar de aprovechar un canal de transmisión que permite llegar a nuevo público objetivo, tal y como lo definen Fenoll y CanoOrón (2017).

Queda enfocado y acotado el objeto de estudio del presente artículo a partir del uso de la información en redes sociales, y cómo esta influye en las campañas electorales de los diferentes partidos políticos. Como apunta Caldevilla-Domínguez (2010):

Un buen uso de las redes sociales puede ayudar, en primer lugar, a cientos de profesionales a conseguir promoción gratuita para nuevos productos y tendencias (Marketing viral), $y$, en segundo lugar, a afianzar a muchas empresas para mejorar su posicionamiento e imagen de marca allende los métodos tradicionales de publicidad. (p. 66)

Dentro del nivel corporativo se pueden distinguir diferentes tipos de entidades, puesto que quedan englobadas las empresas, las administraciones y todas las organizaciones del tercer sector. Todas ellas han transitado el camino hacia la transformación de su comunicación, incorporando las redes sociales como un punto de partida y de llegada hacia sus consumidores, clientes, usuarios y votantes. Esto implica que también los partidos políticos han tenido que reenfocar sus tradicionales comunicaciones para establecer una relación directa con el ciudadano (sea votante o simpatizante), eliminando la figura del intermediario.

Por lo tanto, la transparencia pasa a ser el común denominador de las comunicaciones de los partidos políticos, como del resto de entidades. Si no de forma efectiva al menos aparente, las organizaciones políticas deben establecer este hecho como un objetivo corporativo que tenga su traducción estratégica en sus comunicaciones, incluidas las redes sociales. La consecuencia directa es el factor objetivable que la transparencia posee per se, lo que ha derivado en toda una serie de investigaciones científicas, tanto de tipo cuantitativo como cualitativo, en las que se estudian los intereses de los diferentes intervinientes en el proceso de comunicación para, posteriormente, evaluar sus resultados en aras de mejorar el sistema democrático existente en España (Cabezuelo-Lorenzo et al., 2016). 
La proliferación de canales, soportes y plataformas ha modificado el marco relacional para que sean las organizaciones políticas las que, de forma proactiva, busquen e incluso soliciten la retroalimentación de sus usuarios. Con la congénita asunción de determinados riesgos comunicacionales, los partidos políticos se ven abocados a la movilización de sus usuarios e incluso, como ya se advertía con anterioridad, al diálogo con los mismos para la reflexión o solución de los temas y de las cuestiones que les ocupan y les preocupan. En este punto, se ha incrementado el nivel de interacción y diálogo de las organizaciones con sus usuarios, y de estos últimos entre sí, alcanzando cotas de expansión nunca antes vistas, sobre todo por el desarrollo de las distintas aplicaciones, que, en el entorno omnicanal en el que se desenvuelve la sociedad, dotan de cuasi ubicuidad a los diferentes actores de este panorama, haciendo que las necesidades cuantitativas respecto a alcanzar públicos se hayan facilitado hasta el punto de poder prestar atención a las cualitativas, esto es, los aspectos emocionales del mensaje, cuya atención tiene implicaciones serias y no necesariamente positivas — como advierten Bustos et al. (2019) o Blanco et al. (2019)_, pero útiles, como señala García-Gómez (2014) y Barrientos-Báez et al. (2019).

Volviendo a Casero (2018) para enmarcar correctamente la investigación:

Esta circunstancia está generando la aparición de nuevas formas de comportamiento entre el público a la hora de consumir la información política. En primer lugar, el acceso a este tipo de contenido es breve e ininterrumpido y se produce en cualquier momento y cualquier lugar. (p. 968)

\section{Comunicación política y medición social}

Se da un paso más en la construcción del presente marco teórico, puesto que hay que afrontar la medición como una de las características más ponderables de las redes sociales. La generación de impactos comunicacionales, dadas las características de la Web 2.0, facilita una multitud de interacciones que desata la reflexión acerca de su recopilación, su análisis y su posterior reflexión. El primer paso genera, de forma necesaria, una amalgama de datos, tanto estructurados como no estructurados, que se pueden tratar a través de diferentes herramientas (Villars et al., 2011).

La medición, gracias a las altas capacidades de las herramientas web, puede realizarse tanto en tiempo real como pretérito, lo que ayuda a gestionar los datos, a depurar sus errores, a limitar la investigación de una forma razonada, pragmática y verosímil y a interpretarlos correctamente.

La comunicación política, y en concreto las campañas electorales, no escapa a esta posibilidad que ofrece la medición de los distintos perfiles en medios sociales, teniendo además en cuenta el carácter público de los datos que exponen estas organizaciones, tanto a nivel de contenidos como de interacciones. La ingente cantidad de datos expuesta se transforma en un arma, tanto para los ciudadanos y los investigadores como para las propias organizaciones que utilizan estos canales para lograr sus objetivos (Serrano-Cobos, 2014). 
Muchos autores consideran, en este sentido, que los medios sociales han cambiado la comunicación de los partidos políticos en las últimas campañas electorales (Enguix, 2017; Rodríguez-Andrés, 2016; Ballesteros, 2017; González-Bustamante y Barría, 2019; Fontenla-Pedreira et al., 2020), hasta el punto de que la medición se convierte en argumento metacomunicativo para que los partidos populistas aumenten su compromiso o su afinidad de elección (Gerbaudo, 2018; Engesser et al., 2017).

\section{Diseño y método}

El enfoque metodológico escogido se enmarca en el llamado análisis de redes sociales (ARS). Se utiliza con asiduidad dentro de las ciencias sociales para analizar interacciones y contenidos que se transfieren a través de las aplicaciones. Eligiendo la adecuada, se puede decidir qué aspecto de esa inabarcable realidad nos interesa investigar. Además, ayuda a presentar datos ingentes de información de forma clara y entendible (Sandulescu, 2017). Es, por tanto, necesario cribar el universo de información, y el ARS viene a excluir su contenido redundante, desfasado o trivial (DalleMulle y Davenport, 2017).

El diseño de la investigación propicia un gran número de datos que, sin embargo, tiene unos límites muy claros y que vienen definidos temporalmente por la duración legal de las campañas electorales en España. Los periodos de investigación, por lo tanto, son los siguientes:

1. Del 12 al 28 de abril de 2019.

2. Del 10 al 26 de mayo de 2019.

3. Del 1 al 10 de noviembre de 2019.

Además de la campańa electoral, se han incluido en la investigación el día de reflexión, que tradicionalmente se caracteriza por la ausencia de mensajes, por su interés de cara a comprobar si, en términos efectivos, se respeta esa liturgia política. También se ha incorporado la jornada electoral, por la importancia intrínseca que posee en cuanto a la comunicación política según los diferentes escenarios a los que tienen que hacer frente las distintas opciones políticas.

De igual forma, se acota la investigación a los partidos políticos que están presentes tanto en todas las elecciones como en todas las circunscripciones electorales, cualesquiera que sean los comicios. De esta forma fueron un total de cinco partidos los que entraron en el estudio:

1. Partido Socialista Obrero Español (PSOE).

2. Partido Popular (PP).

3. Unidas Podemos (UP).

4. Vox.

5. Ciudadanos (Cs). 
No es posible realizar el diseño metodológico sin nombrar el análisis de redes sociales de una manera general. Según Pérez Tornero (2015), las redes sociales ofrecen potencialidades en diferentes escenarios, incluidas las campańas electorales. Los perfiles en redes sociales dejan mensajes dirigidos a diferentes públicos, como los que se han observado en el presente estudio, que provocan un diálogo constante y cambiante a través de las diferentes interacciones que también forman parte de esta investigación y que van a variar según la red social:

1. Facebook:
a) Reacciones.
b) Comentarios.
c) Compartidos.

2. Twitter:
a) Me gusta.
b) Retuits.

3. Instagram:
a) Me gusta.
b) Comentarios.
c) Engagement.

Para la medición de estos resultados se puede elegir entre un gran número de aplicaciones que sirven para recoger, extraer y analizar los datos procedentes de estas tres redes sociales. En este caso se escoge Metricool como la más adecuada, por gozar de un protocolo adecuado para los objetivos marcados, aunque supone una limitación metodológica, puesto que en el caso de Instagram solo es posible recoger los datos anteriores de las cuentas business. En el caso de Podemos el paso de una cuenta de tipo personal a una cuenta corporativa no se produjo hasta mitad de noviembre de 2019, con lo que los datos extraídos han sido solo parciales en cuanto al número de seguidores, pero completos en la recogida de las categorías de interacciones que se acaban de reseñar.

Para la fórmula del engagement se tenía que decidir cuál era la oportuna, puesto que este término «es algo más complejo que generar un Me Gusta o Retuits [...] Los mercadólogos deben tener claras las percepciones de sus consumidores, sus deseos, necesidades y preferencias, conectarse con ellos en sus propios términos» (Sánchez Torres y Restrepo, 2015: 45). De entre todas las fórmulas que se pueden encontrar, la que se utiliza es la ratio de engagement como el número de interacciones recibidas en relación con el número de publicaciones. A continuación se desgranan las diferentes tipologías de interacciones en función de cada red social:

1. Facebook:

a) Reacciones.

b) Comentarios.

c) Compartido. 
2. Twitter:
a) Me gusta.
b) Retuits.

3. Instagram:

a) Me gusta.

b) Comentarios.

La medición de los usuarios que acumulan los perfiles de las formaciones políticas y sus variaciones cuantitativas también proporciona interesantes datos y reflexiones. Los periodos sobre los que se ha establecido la comparación han sido los anteriores a los del estudio, con la misma longitud temporal. Esto es, los diecisiete días anteriores a las campañas de abril y mayo, y los diez días anteriores a la campaña de noviembre:

1. Del 26 de marzo al 11 de abril de 2019.

2. Del 23 de abril al 9 de mayo de 2019.

3. Del 22 al 31 de octubre de 2019.

Como se puede observar, y dada la alta intensidad electoral en Espańa en 2019, uno de los periodos de control coincide con otro periodo electoral, concretamente cinco días entre el 23 y el 28 de abril. Esta circunstancia, lejos de ser un impedimento, constituye una fantástica oportunidad para dotar a este estudio de la complejidad necesaria que vivió la propia sociedad española a lo largo de casi diez meses.

No cabe duda de que la medición de los resultados en redes sociales ha supuesto y supone también hoy en día una verdadera obsesión, y por supuesto se incluyen en esta reflexión a los perfiles en redes sociales de los partidos políticos. Pero no es menos cierto que los usuarios de la Web 2.0 también son conscientes de esta circunstancia en la mayoría de los casos, puesto que, con el paso del tiempo y de los acontecimientos, ya son maduros en el uso y las consecuencias de las plataformas de comunicación en línea.

\section{Trabajo de campo, análisis de datos y resultados}

En este punto se realiza un análisis de los datos que se han recogido de los diferentes perfiles de los partidos políticos españoles durante las campañas electorales en 2019. El primero: el número de seguidores y de fanes acumulados en las distintas campañas.

Como se puede observar, Unidas Podemos capitaliza los seguidores en esta red social, al igual que lo hace en Twitter, como se verá posteriormente. La página de seguidores acumula más fanes en cualquiera de los tres puntos de medición $(1.251 .000,1.251 .967$ y 1.264 .808$)$ que el resto de las formaciones políticas en su mejor momento (Vox: 358.688, Ciudadanos: 338.330, Partido Popular: 210.991 y Partido Socialista Obrero Español: 188.578. Total: 1.096.587). 
Figura 1. Acumulado de fanes en Facebook

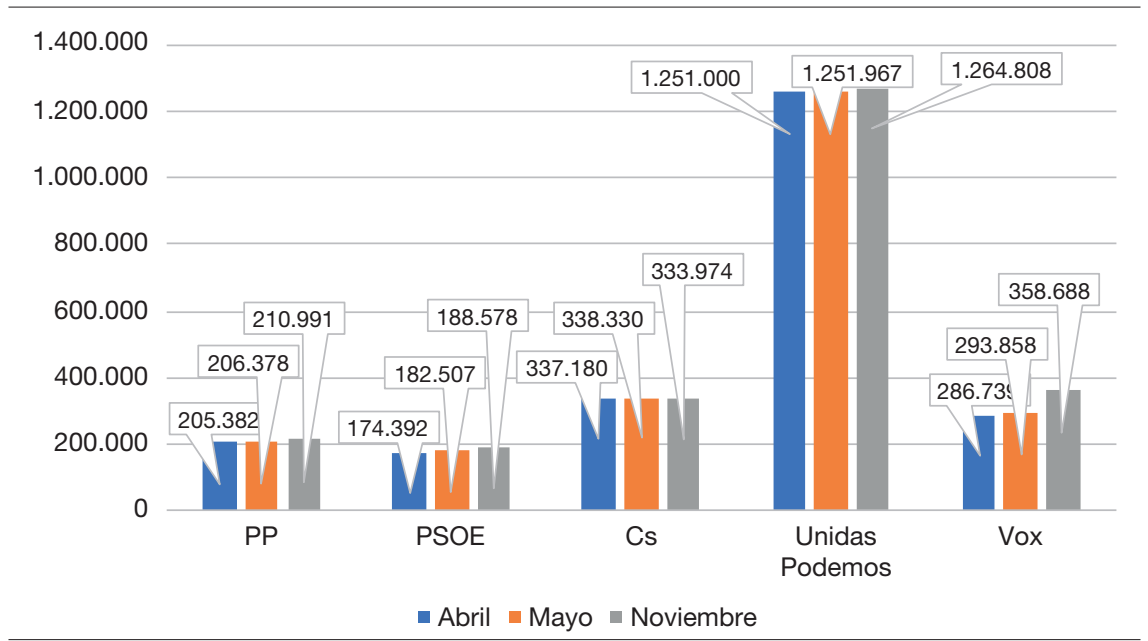

Fuente: elaboración propia y Metricool.

Sin embargo, el partido político liderado por Pablo Iglesias no es el que ha acumulado un mayor porcentaje de crecimiento. Si lo observamos en las elecciones celebradas en abril, Vox incrementa su número de seguidores en un $7,17 \%$, por lo que constituye el volumen más alto en el periodo de observación. En las elecciones de mayo, es el PSOE el que más sube, con un 2,53\%, $\mathrm{y}$ en las de noviembre vuelve a ser el partido dirigido por Santiago Abascal el que ostenta un mayor incremento, con un $3,74 \%$.

Si excluimos los periodos de control y solo tenemos en cuenta los datos recogidos en las distintas campañas, el incremento en términos absolutos más importante en cuanto a porcentaje de seguidores vuelve a ser el de Vox, con un $25,09 \%$, mientras que el peor parado es Ciudadanos, que acumula pérdidas de seguidores y porcentuales, con un $-0,95 \%$.

$\mathrm{Al}$ analizar esta segunda red social se encuentran resultados dispares con respecto a los de Facebook. Vuelve a ser Unidas Podemos la formación con una mayor acumulación de seguidores en su perfil, aunque ya no de forma tan absoluta como en la red creada por Mark Zuckerberg. En la segunda posición se sitúa el Partido Popular en lugar de Ciudadanos, y Vox queda relegada a una última posición, con bastante distancia en las elecciones de abril y mayo, aunque recorta algo de distancia en las elecciones generales del 10 de noviembre de 2019.

Ningún partido pierde seguidores en ninguna cita electoral, a excepción de la formación presidida entonces por Albert Rivera, que, al igual que en Facebook, cae en términos absolutos desde la primera llamada a las urnas en 2019 , con un $-0,57 \%$. Con respecto a su periodo de control en noviembre sube un $0,13 \%$, pero esta cifra es la más pequeña de todos los partidos en ese periodo. 
Figura 2. Acumulado de seguidores en Twitter

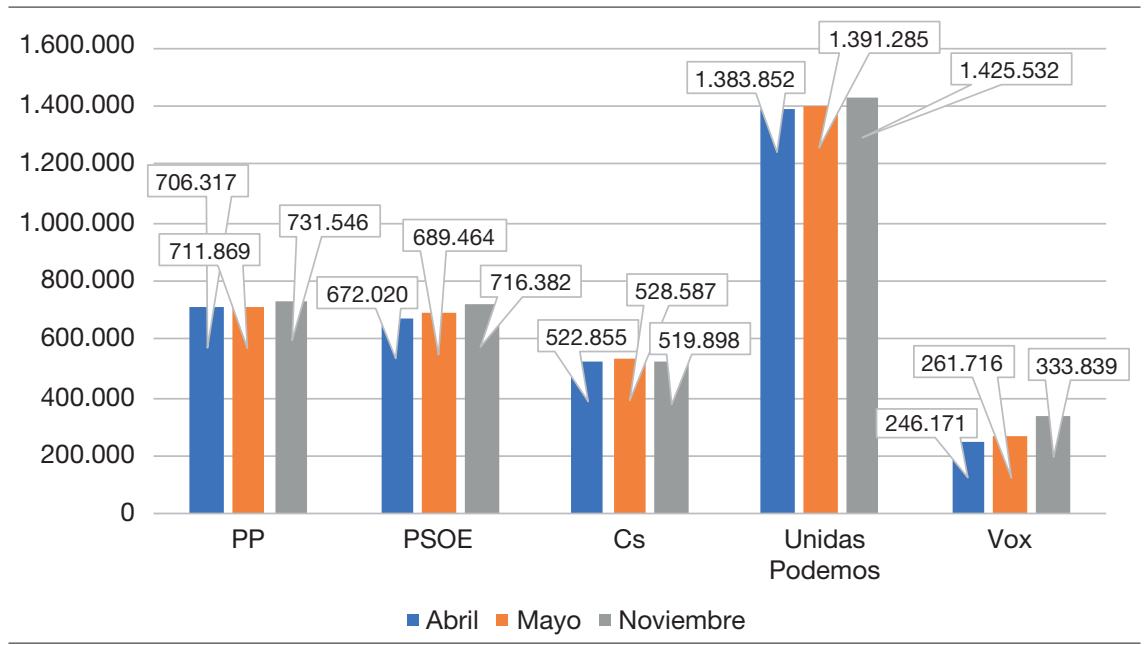

Fuente: elaboración propia y Metricool.

Vuelve a ser remarcable el progreso de Vox en esta red social, puesto que, a pesar de que se ha destacado que acumula menos seguidores que el resto de los partidos de forma considerable, es la formación que mayores incrementos ha recogido en toda la investigación. Teniendo en cuenta los tres periodos de control, Vox acumula las mayores subidas en todos (abril: un 9,37\%; mayo: un $1,45 \%$, y noviembre: un 5,06\%) y dos de las tres mayores en todas las citas y con todos los partidos, quedando en segunda posición la subida del 2,53\% de Ciudadanos en abril.

En términos absolutos, es decir, teniendo en cuenta solo los datos de los periodos de investigación, la mayor subida también es de Vox (un 35,61\%), seguido a mucha distancia del PSOE (un 6,6\%).

En esta última red analizada se pueden volver a observar datos realmente curiosos, si bien limitados, puesto que el análisis de Podemos, como ya se señaló en el apartado de metodología, viene condicionado por no haber cambiado su cuenta a business hasta mitad de noviembre de 2019. Esta circunstancia hace que la cifra señalada en las elecciones de noviembre no sea real, pero, al solo haber transcurrido cinco días, sí que puede ser muy aproximada.

Es Vox el que acumula un mayor número de seguidores con casi la misma circunstancia que la de Podemos en Facebook, esto es, en los mejores datos del PSOE, PP, Cs y UP superan por poco juntos (481.583) al mejor dato de Vox (438.148), con algo menos de cincuenta mil seguidores. De igual forma, la formación de Santiago Abascal es la que registra un mayor incremento en términos absolutos, puesto que desde abril hasta noviembre aumenta sus seguidores en un $51,22 \%$. 
Figura 3. Acumulado de seguidores en Instagram

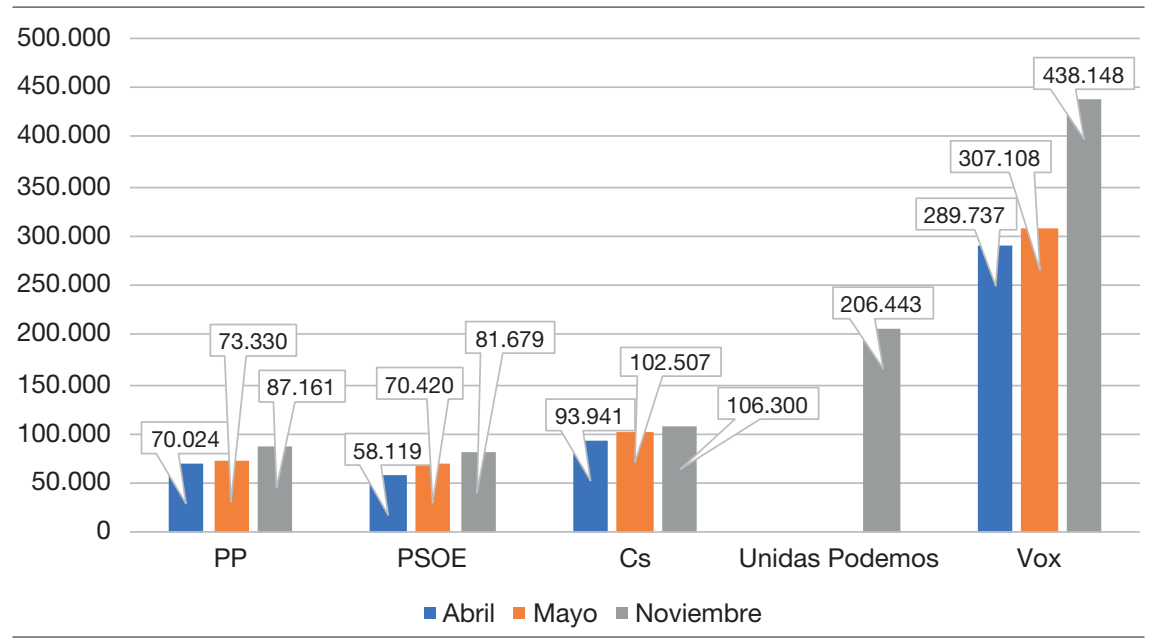

Fuente: elaboración propia y Metricool.

Figura 4. Agrupación de publicaciones según el partido político, la red social y la campaña electoral

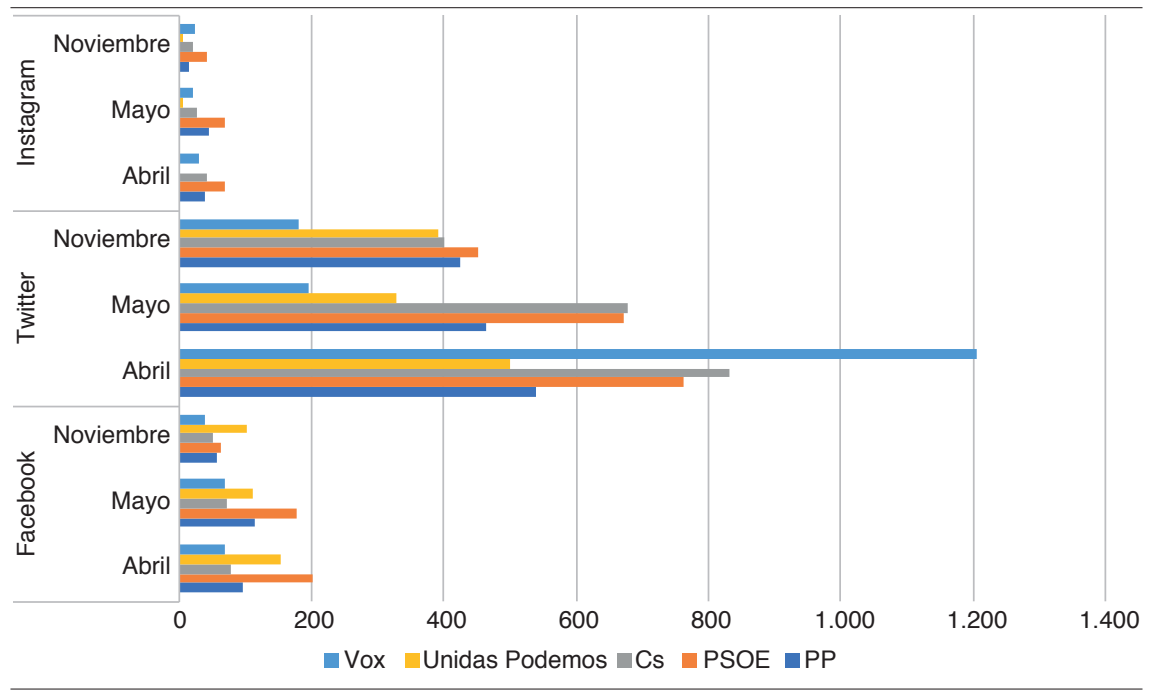

Fuente: elaboración propia y Metricool.

Sin embargo, valorando cada fecha electoral por separado, es el PSOE el que experimenta una mayor subida en abril (un 30,15\%), al igual que en mayo (un 7,3\%), mientras que en noviembre es Vox el que tiene un mayor incremento, con un 5,06. Si afrontamos las cifras de forma independiente en el momento en que se produjeron, el PSOE con su subida de abril ocupa la 
primera posición (un 30,15\%). También en abril se producen el segundo y el tercer mayores aumentos, con el porcentaje de Vox (un 22,91\%) y el de Ciudadanos (un 22,54\%), dejando claro que esta primera cita de 2019 fue la que supuso un gran cambio en la política de comunicación de los partidos políticos en esta red social.

Ahora se evalúa otro aspecto importante del análisis de las redes sociales de los partidos políticos en las campañas electorales de 2019, en este caso las publicaciones. Se presentan en este gráfico de forma agrupada teniendo en cuenta la red social y la campańa electoral puesto que, de esta forma, resulta evidente visualmente lo que los datos nos trasladan. Nos enfrentamos a casi diez mil impactos comunicativos (9.938) distribuidos por redes sociales de la siguiente forma:

1. Facebook: 1.451.

2. Twitter: 8.208 .

3. Instagram: 459.

Los partidos políticos distribuyeron el total de sus mensajes de la siguiente forma:

1. PSOE: 2.507 .

2. PP: 1.800 .

3. Unidas Podemos: 1.597.

4. Ciudadanos: 2.198 .

5. Vox: 1.836.

Y en cuanto a campañas electorales, los mensajes acumulados fueron:

1. Abril: 4.618 .

2. Mayo: 3.050 .

3. Noviembre: 2.270 .

En primer lugar, queda claro que Twitter es la red social que mayor cifra de impactos emite. El acumulado con menor número de tuits, que se produce en las elecciones de noviembre (1.849), supera el sumatorio de todas las entradas en Facebook en el conjunto de las campañas (1.451) y multiplica por cuatro el de las publicaciones en Instagram (459). Las particularidades de esta red social, sin lugar a dudas, han llevado a esta circunstancia, especialmente la inmediatez para transmitir la información.

En segundo lugar, la importancia de la cita electoral de abril queda patente por el volumen de mensajes emitidos (4.618), comparada especialmente con la cita de mayo (3.050). La comparación no se puede efectuar de la misma forma con la campaña del mes de noviembre (2.270), puesto que esta se vio recortada en una semana, aunque es fácil extrapolarla y observar cómo el volumen alcanzaría unas cifras similares. 
En este sentido, y cruzando ahora la red social con los comicios electorales, es Twitter en abril la que denota más publicaciones (3.841) y es Vox el partido que mayor esfuerzo cuantitativo realiza, con 1.207 impactos. Esta cifra no solo es la más alta de toda la investigación, sino que está seguida de la de Ciudadanos (831) y del PSOE (763) en la misma red social y en las mismas fechas, como los tres primeros registros del estudio.

En cuanto a partidos se refiere, el PSOE es el que emite un mayor número de mensajes (2.597). Su regularidad es manifiesta en el gráfico, donde no abandona en ninguna cita electoral ni en ninguna red social alguna de las tres primeras posiciones en cuanto a publicaciones.

1. Es primero en cinco ocasiones:
a) Facebook (abril y mayo).
b) Twitter (noviembre).
c) Instagram (abril y mayo).

2. Segundo dos veces:
a) Facebook (noviembre).
b) Twitter (mayo).

3. Y tercero en otras dos:
a) Twitter (abril).
b) Instagram (noviembre).

Figura 5. Engagement Facebook

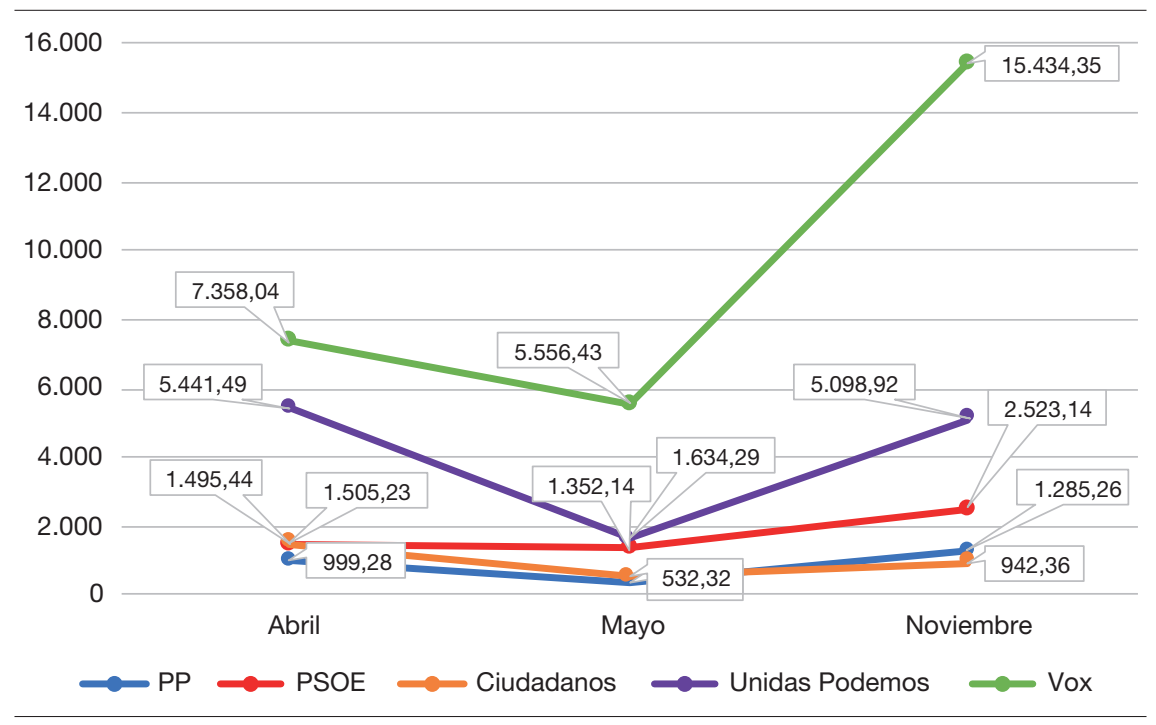

Fuente: elaboración propia y Metricool.

El último aspecto que se tiene en cuenta en el análisis va a ser el engagement, revisándolo por cada red social observada. Tomamos como primera 
referencia Facebook, donde podemos señalar el claro liderazgo que posee Vox frente al resto de partidos, especialmente en las elecciones generales del 10 de noviembre de 2019. El partido de Santiago Abascal acumuló 617.374 interacciones, de las que 445.018 pertenecen a reacciones; 30.987 , a comentarios, y 141.369, a entradas compartidas. Las cifras de interactuación se alzan con la primera posición de todas las formaciones en todas las elecciones, y solo Unidas Podemos alcanza una cifra que está por debajo de los dos mil puntos en la cita de abril.

En cuanto a la evolución de los partidos a lo largo de las tres elecciones, todos los partidos vieron reducido su engagement en las elecciones del mes de mayo, sumando entre todos $9.481,2$, prácticamente la mitad que en el mes de abril $(16.799,48)$ y casi tres veces menos que el de noviembre $(25.284,04)$. La caída más pronunciada es la de Unidas Podemos, con cerca de un setenta por ciento $(-69,97 \%)$, seguido de cerca por Ciudadanos $(-64,4 \%)$ y el Partido Popular $(-59,37 \%)$.

Las elecciones de noviembre muestran resultados dispares. Por un lado, la comparativa entre mayo y noviembre deja al Partido Popular (un 216,56\%) y a Unidas Podemos (un 212\%) como las formaciones con un mayor incremento de su engagement. Todas las organizaciones políticas experimentan un fuerte aumento en esta comparativa, aunque los datos cambian si el periodo que comparamos es el de las dos elecciones generales, es decir, las de abril y las de noviembre.

En este caso la mayor subida es la de Vox (un 109,76\%) con más del doble del engagement al final del estudio que al principio. Muy lejos queda el PSOE (un 67,63\%), aunque su ascenso se sitúa por encima de los dos tercios, lo que a su vez supone más de un tercio más que lo que sube el Partido Popular (un 28,62\%). Las cifras de Ciudadanos y Unidas Podemos se sitúan en cifras negativas, siendo la organización liderada por Pablo Iglesias la que menos cae $(-6,3 \%)$, mientras que la formación naranja desciende más de un tercio $(-36,98 \%)$, lo que deja su cifra por debajo de los mil puntos.

Los resultados de Twitter presentan coincidencias y discrepancias con respecto a los de Facebook. Se puede observar que la ratio del engagement es mucho menor, dado que el volumen de publicación es mucho más alto. Además, las cifras absolutas de Vox ya no son las mejores en todas las citas electorales, puesto que Unidas Podemos $(1.301,84)$ consigue situarse en la primera plaza en la llamada a las urnas del mes de abril de 2019.

También se puede seńalar otro descenso generalizado de la ratio en las elecciones de mayo de todas las formaciones salvo de Vox (un 274,68\%), que casi triplica su resultado anterior. Dentro de los partidos que bajan, el que menos lo hace es el Partido Socialista Obrero Español, que pierde casi un treinta por ciento $(-29,67 \%)$ de engagement. A pesar de la bajada global, el fuerte incremento de Vox hace que el engagement en cifras absolutas sea mayor en mayo $(3.552,41)$ que en abril $(3.260,38)$. 
Figura 6. Engagement Twitter

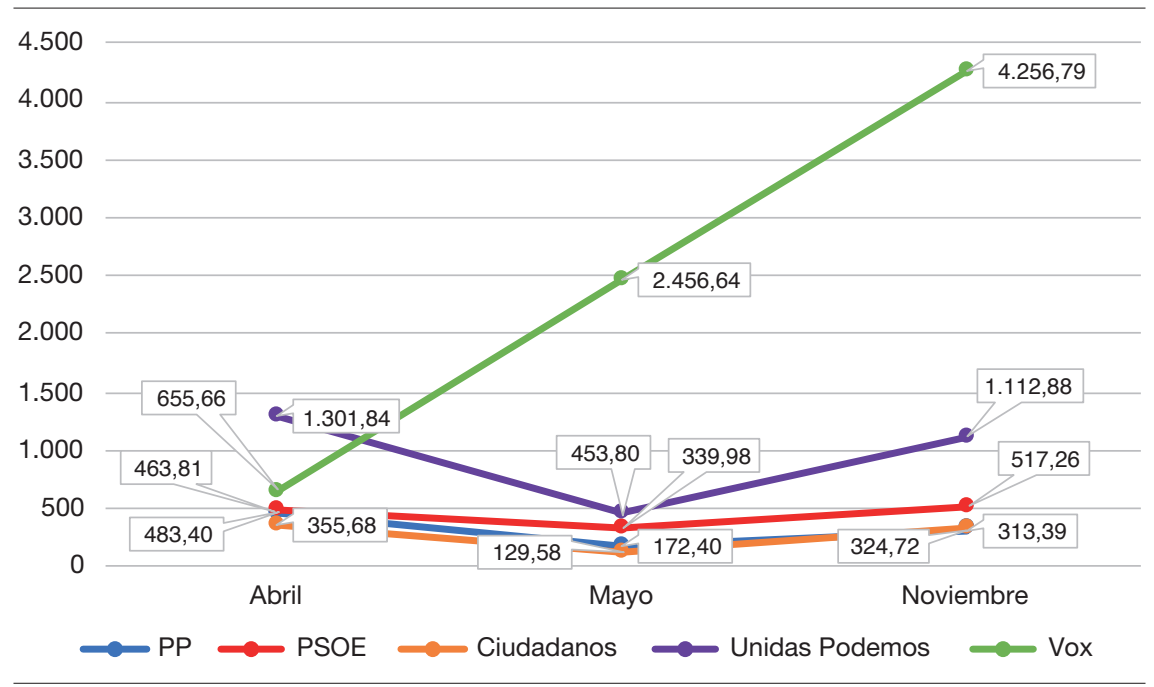

Fuente: elaboración propia y Metricool.

Vox además encadena dos incrementos consecutivos, al contrario de lo que ocurría en Facebook, siendo el primero de los mismos el más fuerte, es decir, entre el mes de abril y el de mayo. Sin embargo, si se tiene en cuenta todo el periodo estudiado eliminando el periodo intermedio, la cifra es mucho más pronunciada (un 549,24\%) para la formación verde, que deja muy lejos el porcentaje de subida del PSOE (un 7\%). El resto de los partidos acumulan pérdidas en su engagement: Ciudadanos baja un $8,7 \%$, Unidas Podemos se deja un $14,51 \%$ y el Partido Popular se queda en casi un tercio negativo $(-32,43 \%)$. Igualmente, el volumen de interacciones recogidas por Vox es más alto que en ningún otro partido, con un pico máximo en noviembre de 770.479 , de las cuales 530.897 son «me gusta» y 239.582, retuits. En cuanto a las mejores posiciones en la ratio del engagement llama la atención que ni el Partido Popular ni Ciudadanos logran ninguna de las tres primeras plazas en ninguna de las citas electorales.

El último gráfico muestra el análisis del engagement en Instagram, donde las cifras de Vox se pueden calificar de abrumadoras. La formación de Abascal está muy por encima de cualquiera de los otros partidos políticos en todos los periodos estudiados. Su peor cifra $(26.951,23)$ es más que la suma de las tres mejores cifras del resto de formaciones $(24.583,84)$ :

1. Partido Popular en noviembre: $3.628,5$.

2. PSOE en mayo: 3015,66 .

3. Ciudadanos en abril: $3.976,68$.

4. Unidas Podemos en noviembre: 13.963. 
Figura 7. Engagement Instagram

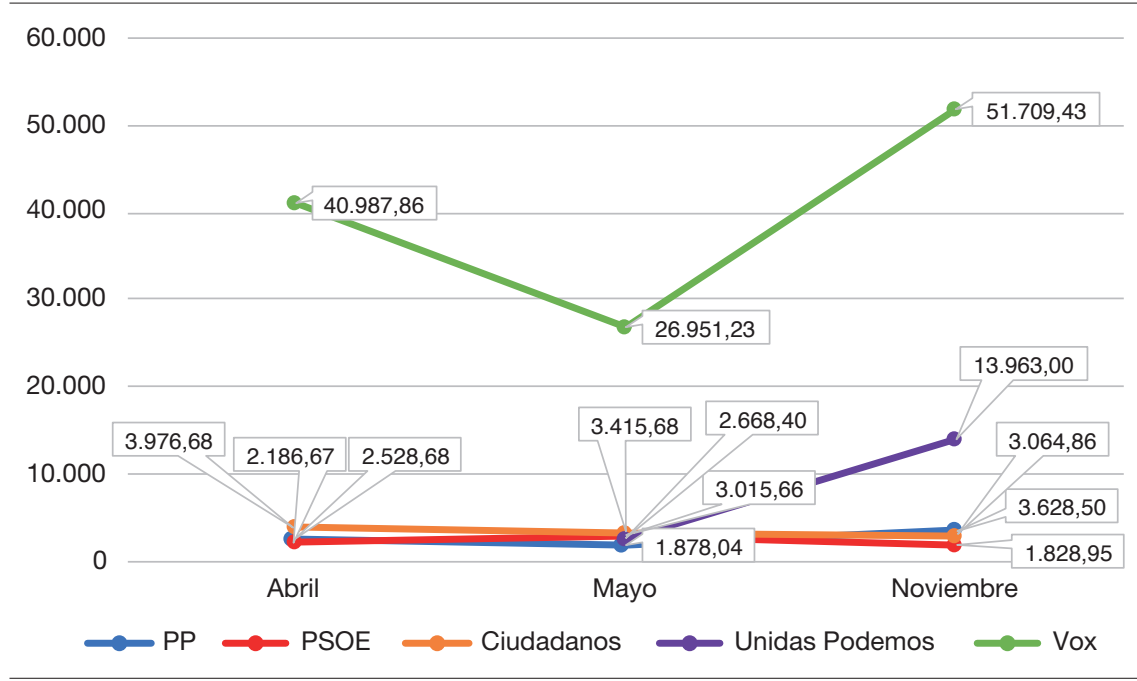

Fuente: elaboración propia y Metricool.

Es de nuevo en noviembre cuando Vox registra sus mejores números en cuanto a interacciones, con más de un millón (1.189.317). Los «me gusta» arrasan con 1.147.979, mientras que los comentarios suman 41.338. El resto de los partidos se quedan muy lejos de estos guarismos, de hecho, el segundo mejor registro en cuanto a interacciones es el del PSOE en mayo (205.065) y el tercero es el de Ciudadanos en abril (163.044).

Todos los partidos vuelven a caer en el engagement entre las citas de abril y mayo, a excepción del PSOE, que lo incrementa en un 37,91\%. Es Vox el que más cae, descendiendo un 34,25\%, y el que menos lo hace es Ciudadanos, con una bajada del 14,11\%. En la comparación entre las elecciones de mayo y noviembre observamos un astronómico ascenso de Unidas Podemos (un 423,27\%), mientras que el Partido Popular (un 93,21\%) y Vox (un $91,86)$ también experimentan un gran ascenso que casi les hace doblar sus ratios. Ciudadanos cae un 10,27\% y el descenso más agudo es el del PSOE, con una bajada del 39,35\%. Por último, comparando las dos citas de abril y noviembre, el PP aumenta casi un cincuenta por ciento (un 43,49\%), seguido de Vox, con más de un cuarto de subida (un 26,16\%). El resto de las formaciones entran en cifras negativas, con el PSOE $(-16,36 \%)$ y Ciudadanos $(-22,93 \%)$ abanderando estas malas noticias para su engagement, mientras que Podemos se queda en el limbo, al no haber podido registrar sus datos.

\section{Conclusiones y discusión}

Se aborda en primer lugar el objetivo general de la investigación, que era conocer el uso de las redes sociales por parte de los partidos políticos en las 
tres campañas electorales que hubo en España en el año 2019. Por número de seguidores, por publicaciones y por su ratio del engagement, es Vox el que mejor se ha manejado en Facebook, Twitter e Instagram durante este extenso periodo de estudio, puesto que alcanza los mejores guarismos en todas las redes, especialmente en los datos del engagement.

Desgranando ahora cada aspecto que se ha analizado, queda patente que por número de seguidores es Unidas Podemos la formación que consigue un mayor éxito, encabezando dos de las tres redes: Facebook y Twitter. Instagram se tiñe de verde en cada aspecto analizado. Vox encuentra en esta plataforma su terreno donde abonar y lo aprovecha consiguiendo más seguidores que ningún otro partido político y publicando en un volumen conveniente para recoger las interacciones de estos mismos acólitos, lo que genera unas cifras de engagement imbatibles para cualquier red y para cualquier partido.

De forma general, se produce un estancamiento en las cifras investigadas durante las elecciones municipales, autonómicas y europeas celebradas el 28 de abril de 2019. Si bien es cierto que los seguidores y el número de publicaciones de todas las redes experimentan un ligero aumento, el engagement se reduce en una proporción mucho más grande debido a la proximidad de ambas campañas y el cansancio que en el elector produjo esta circunstancia.

Si se atiende ahora a la parte de publicaciones, es Twitter la red social que se muestra como el canal favorito y el más consolidado entre los partidos políticos para emitir impactos comunicacionales. Su inmediatez ayuda a que haya una mayor cantidad de mensajes, complementando aquellos que se trasladan desde las otras dos estudiadas: Facebook e Instagram. Por lo tanto, las formaciones encuentran esta presencia ubicua en redes sociales como una característica complementaria para la difusión de sus mensajes y no como sustitutiva de unas plataformas por otras.

Reflexionando ahora sobre el engagement, se puede concluir que es Instagram la que genera mejores números, teniendo en cuenta la fórmula elegida que pone en relación el total de interacciones con la cantidad de publicaciones. Precisamente engarzando esta circunstancia con la conclusión anterior, se observa como Twitter es la red que presenta peores números. El hecho de que Instagram pertenezca a Facebook y las posibles facilidades para la vinculación de contenidos entre ambas provoca que Facebook presente unas cifras muy buenas, lo que la consolida como una referencia.

A tenor de los resultados establecidos, son Vox y Unidas Podemos los partidos más ciberactivos y que más movilización producen a través de las redes sociales. Copan los primeros dos puestos en la mayoría de los análisis, aunque llama la atención que Vox cae mucho en cuanto a seguidores en Twitter y, como se ha señalado, en Instagram sin embargo despunta sobremanera. Esto no hace sino confirmar que poseen una mayor afinidad de elección (Gerbaudo, 2018), puesto que explotan una narrativa rebelde junto a un desarrollo continuo de las redes sociales, lo que les hace asumir la voz de los decepcionados con el sistema para identificarse con ellos mediante estos canales. 
En este punto la investigación afronta sus límites, puesto que un desarrollo cualitativo nos ayudaría a proporcionar una visión holística del objeto de investigación. Sin embargo, este punto solo puede ser de inflexión para futuras investigaciones sobre partidos populistas y campańas electorales, donde la comparación con citas de otros países también deban ponerse bajo el foco y aprovechar de esta forma la rica literatura existente (Cervi y Roca, 2017; Bastos y Farkas, 2019; Cervi, 2020; Recuero et al., 2020).

Los resultados del bipartidismo tradicional español son bastante estables en todas las redes y en todas las características analizadas, y es Ciudadanos el partido que más desciende, sobre todo teniendo en cuenta el inicio y el fin de esta investigación, al igual que cuando se establecen periodos de control análogos a las campañas electorales pero anteriores temporalmente. La formación naranja disminuye en todas las características analizadas y su repercusión en las urnas en la cita de noviembre quedó patente.

Por todo ello se concluye que las redes sociales se han consolidado como canales de comunicación para los partidos políticos en la difusión de sus mensajes. Las distintas formaciones aprovechan las potencialidades de la Web 2.0 para la producción de contenidos, la interacción con los usuarios, la captación de nuevos seguidores y la fidelización de sus acólitos. Los partidos saben que sus mensajes en campańa electoral van a ser escuchados y escudrińados, y aprovechan este momento álgido para buscar en las redes sociales un punto de apoyo más en su estrategia de comunicación que traiga, si es posible, los mejores resultados posibles en las urnas.

\section{Referencias bibliográficas}

BAllesteros Herencia, C. A. (2017). Movilización en las campañas electorales a través de las redes sociales: Las elecciones generales españolas de 2015. Conference: International Association for Media and Communication Research (IAMCR). Cartagena de Indias (Colombia). Recuperado de <https://bit.ly/39VoBSe $>$.

BARrientos-BÁez, A.; BARquero-CABrero, M. y RodrígueZ-Terceño, J. (2019). «Educación emocional como contenido transversal para una nueva política educativa: el caso del Grado de Turismo». Revista Utopía y Praxis Latinoamericana, 24 (4), 147-165. Recuperado de <https://produccioncientificaluz.org/ index.php/utopia/article/view/29796>.

Barrientos-BÁEZ, A.; CALdeVilla-DomíngueZ, D. y VArgas-Delgado, J. J. (2019). «El protocolo, la puesta en escena y la persuasión en los debates políticos televisados». Redmarka: Revista de Marketing Aplicado, 23 (3), 17-27. <https://doi.org/10.17979/redma.2019.23.3.5872>

BAstos, M. y FARKAS, J. (2019). «Donald Trump Is My President!: The Internet Research Agency Propaganda Machine». Social Media + Society, 1-13. <https://doi.org/10.1177/2056305119865466>

Blanco Alfonso, I.; García Galera, C. y Tejedor Calvo, S. (2019). «El impacto de las fake news en la investigación en Ciencias Sociales: Revisión bibliográfica sistematizada». Historia y Comunicación Social, 24 (2), 449-469.

<https://doi.org/10.5209/hics.66290> 
Bustos Martínez, L.; Santiago Ortega, P. P. de; Martínez Miró, M. Á. y RENGifo HidAlgo, M. S. (2019). «Discursos de odio: una epidemia que se propaga en la red. Estado de la cuestión sobre el racismo y la xenofobia en las redes sociales». Mediaciones Sociales, 18, 25-42. $<$ https://doi.org/10.5209/meso.64527>

Cabezuelo-Lorenzo, F.; Rey-García, P. y Tapia-Frade, A. (2016). "Análisis de las herramientas de control ciudadano sobre los representantes públicos: la transparencia informativa municipal en Castilla y León». Revista Latina de Comunicación Social, 71, 1261-1279. <https://doi.org/10.4185/RLCS-2016-1145>

CaldeVilla-DomíngueZ, D. (2010). «Las redes sociales. Tipología, uso y consumo de las redes 2.0 en la sociedad digital actual» Documentación de las Ciencias de la Información, 33, 45-68. Recuperado de <https://revistas.ucm.es/index.php/ DCIN/article/view/DCIN1010110045A/18656>.

CASERO-RIPOLLÉS, A. (2018). «Research on political information and social media: Key points and challenges for the future». El Profesional de la Información, 27 (5), 964-974. <https://doi.org/10.3145/epi.2018.sep.01>

Catalina-García, B.; López de Ayala-López, M. C. y Martínez Pastor, E. (2019). «Usos comunicativos de las nuevas tecnologías entre los menores: Percepción de sus profesores sobre oportunidades y riesgos digitales». Mediaciones Sociales, 18, 43-57. $<$ https://doi.org/10.5209/meso.64311>

Cervi, L. (2020). "Veni, Vidi, Facebooked-Live: Análisis del éxito de Matteo Salvini en Facebook». Revista CIDOB d'Afers Internacionals, 124, 99-122. <https://doi.org/10.24241/rcai.2020.124.1.99>

CERVI, L. y RoCA, N. (2017). «Cap a l'americanització de les campanyes electorals?: L'ús de Facebook i Twitter a Espanya, Estats Units i Noruega». Anàlisi: Quaderns de Comunicació i Cultura, 56, 87-100. <https://dx.doi.org/10.5565/rev/analisi.3072>

Dallemulle, L. y DAVenport, T. (2017). «What's Your Data Strategy?». Harvard Business Review. Recuperado de <https://hbr.org/2017/05/whats-your-data-strategy> [Fecha de consulta: 10/07/2020].

Engesser, S.; ERnst, N.; Esser, F. y BüChel, F. (2017). «Populism and social media: How politicians spread a fragmented ideology». Information, Communication \& Society, 20 (8), 1109-1126. <https://doi.org/10.1080/1369118X.2016.1207697>

ENGUiX Oliver, S. (2017). "Impacto político e informativo de las redes sociales: Esferas de actuación y comparación con los medios». Anàlisi: Quaderns de Comunicació i Cultura, 56, 71-85. <https://dx.doi.org/10.5565/rev/analisi.3090>

FEIJOO FERnÁNDEZ, B. y GUERRERO, J. J. (2018). «La investigación de mercados en redes sociales: Conoce a tu consumidor por lo que publica en Facebook y Twitter. Análisis de caso de ADT Security Services en Chile». Vivat Academia: Revista de Comunicación, 144, 19-35. <https://doi.org/10.15178/va.2018.144.19-35>

FENOLL, V. y CANO-ORÓN, L. (2017). «Participación ciudadana en los perfiles de Facebook de los partidos españoles: Análisis de comentarios en la campaña electoral de 2015». Communication \& Society, 30 (4), 131-148. Recuperado de <https:// hdl.handle.net/10171/54614>. 
Fontenla-Pedreira, J.; RÚAS-ARAúJo, J. y PUENTES-RIVERA, I. (2020). «El debate electoral de las elecciones generales del $10 \mathrm{~N}$ de 2019 en las redes sociales de RTVE». El Profesional de la Información, 29 (6). <https://doi.org/10.3145/epi.2020.nov.03>

GARCíA Gómez, M. (2014). "Inteligencia emocional: las emociones son útiles». Revista de Ciencias de la Comunicación e Información, 19 (1), 23-36. <https://doi.org/10.35742/revistaccomunicacioneinformacion.2014.19.23-36>

Gerbaudo, P. (2018). "Social media and populism: An elective affinity?». Media, Culture \& Society, 40 (5), 745-753.

GonzÁlez-Bustamante, B. y BARRíA, D. (2019). «Expansión de la esfera pública en Chile: Redes sociales, campañas electorales y participación digital». En: VAlle, N. del (ed.). Transformaciones de la esfera pública en el Chile neoliberal: Luchas sociales, espacio público y pluralismo informativo. Santiago de Chile: RIL Editores.

JAKIWARA GRÁNDEZ, L. G. (2016). «Las habilidades socioemocionales en los jóvenes: una propuesta de desarrollo humano integral». Revista de Ciencias de la Comunicación e Información, 2 (1), 67-81. <https://doi.org/10.35742/rcci.2016.21(2).67-81>

MARTÍNEZ ROLÁN, X. (2018). "La actividad de los partidos políticos espańoles en Facebook 2014-2018: La tiranía del algoritmo». Revista de Comunicación de la SEECI, 47, 143-155.

<https://doi.org/10.15198/seeci.2018.0.143-155>

PÉREZ-TORNERO, J. (2015). «El futuro de la sociedad digital y los nuevos valores de la educación en medios». Comunicar, 25, 247-258. Recuperado de <www.redalyc. org/pdf/158/15825035.pdf>.

Recuero, R.; SoAres, F. B. y GruZD, A. (2020). «Hyperpartisanship, Disinformation and Political Conversations on Twitter: The Brazilian Presidential Election of 2018». Proceedings of the International AAAI Conference on Web and Social Media, 14 (1), 569-578. Recuperado de <https:/www.aaai.org/ojs/index.php/ ICWSM/article/view/7324>.

RODRÍGUEZ ANDRÉS, R. (2016). «Buscando votos “online”: Las campańas electorales de Hillary Clinton en Internet y las redes sociales». En: KAUfMANN, Alicia E. y Crespo, C. (eds.). Hillary: El poder de la superación. Madrid: Catarata, 179193.

SAMUEL-AZRÁN, T. y HAYAT, T. (2019). "Online news recommendations credibility: The tie is mightier than the source». Comunicar, 60, 71-80. <https://doi.org/10.3916/C60-2019-07>

SÁNCHEZ-Torres, W. C. y Restrepo, J. C. (2015). "Marketing en tiempo real y el fortalecimiento del mercadólogo». Escenarios: Empresa y territorio, 4 (4), 33-52. Recuperado de <https://revistas.esumer.edu.co/index.php/escenarios/article/ view/71>.

SANDUlesCu, A. (2017). Fundamentos de métrica digital en ciencias de la comunicación. Barcelona: Editorial UOC.

SANTIAGO, R. y NAVARIDAS, F. (2012). «La web 2.0 en escena: Web 2.0 comes on the scene». Pixel-Bit: Revista de Medios y Educación, 41, 19-30. Recuperado de <https://www.redalyc.org/pdf/368/36828247002.pdf>.

SERRANO-COBOS, J. (2014). "Big data y analítica web: Estudiar las corrientes y pescar en un océano de datos». El Profesional de la Información, 23 (6), 561-565. <https://doi.org/10.3145/epi.2014.nov.01> 
Villars, R. L.; OlOFSON, C. W. y EASTWOOD, M. (2011). Big data: What it is and why you should care. IDC White Paper.

Woolley, J. K.; Limperos, A. M. y Oliver, M. B. (2010). «The 2008 presidential election, 2.0: A content analysis of user-generated political Facebook groups». Mass Communication \& Society, 13 (5), 631-652.

<https://doi.org/10.1080/15205436.2010.516864> 